Running Head: Ego depletion may disappear by 2020

To appear in the special issue on "Ego depletion and self-control: Conceptual and empirical advances" in Social Psychology, edited by Junhua Dang and Martin S. Hagger https://eu.hogrefe.com/fileadmin/user_upload/global/journals/Hogrefe_Publishing/Social_Psychology/zsp_a000335.pdf

\title{
Ego depletion may disappear by 2020
}

\author{
Miguel A. Vadillo \\ Departamento de Psicología Básica, Universidad Autónoma de Madrid, Spain
}

Mailing address:

\author{
Miguel A. Vadillo \\ Departamento de Psicología Básica \\ Facultad de Psicología \\ Universidad Autónoma de Madrid \\ 28049 Madrid, Spain
}

e-mail: miguel.vadillo@uam.es

Author Note: The present work was supported by grants 2016-T1/SOC-1395 from Comunidad de Madrid (Programa de Atracción de Talento Investigador) and PSI201785159-P from Agencia Estatal de Investigación, Ministerio de Economía y Competitividad. I am also indebted to Evan Carter, Marta Ferrero, Tom Hardwicke, David Luque, and David Shanks for their valuable comments on earlier versions of this article. I would like to dedicate this paper to my son, Daniel, who came to this world just in time to see the twilight of ego depletion. Correspondence concerning this article should be addressed to Miguel A. Vadillo, Departamento de Psicología Básica, Facultad de Psicología, Universidad Autónoma de Madrid, 28049 Madrid, Spain. E-mail: miguel.vadillo@uam.es 


\begin{abstract}
Ego depletion has been successfully replicated in hundreds of studies. Yet the most recent large-scale Registered Replication Reports, comprising thousands of participants, have yielded disappointingly small effects, sometimes even failing to reach statistical significance. Although these results may seem surprising, in the present article I suggest that they are perfectly consistent with a long-term decline in the size of the depletion effects that can be traced back to at least 10 years ago, well before any of the Registered Replication Reports on ego depletion were conceived. The decline seems to be at least partly due to a parallel trend towards publishing better and less biased research.
\end{abstract}

Keywords: Decline effects; Ego-depletion; Publication bias; Reporting bias. 
"Francis Bacon, the early-modern philosopher and pioneer of the scientific method, once declared that experiments were essential, because they allowed us to 'put nature to the question.' But it appears that nature often gives us different answers." (Lehrer, 2011, p. 53)

Intuitively, one would expect that a series of scientific studies exploring the same phenomenon should yield consistent results over time. Yet, meta-research reveals that many scientific findings show 'decline effects', whereby initial studies report substantially larger effect sizes than subsequent ones addressing the same question (Ioannidis, 2008; Protzko \& Schooler, 2017; Schooler, 2011; Trikalinos \& Ioannidis, 2005). For instance, in the biomedical domain there is evidence that early randomized controlled trials tend to overestimate the efficacy of treatments (Gehr, Weiss, \& Porzsolt, 2006; Trikalinos, Churchill, Ferri, Leucht, Tuunainen, Wahlbeck, \& Ioannidis, 2004) and that subsequent trials usually yield smaller (and likely more accurate) estimates (Pereira, Horwitz, \& Ioannidis, 2012). Effect sizes in ecology and evolution research seem to follow a similar downward trend (Jennions \& Moller, 2002; Simmons, Tomkins, Kotiaho, \& Hunt, 1999).

In psychology and the behavioral sciences, large-scale replication studies have typically obtained smaller effects than the original experiments they were intended to replicate. In the famous Open Science Collaboration (2015), a massive multi-site study aimed at replicating 100 random studies from four prestigious psychology journals, the effect sizes of the replications were, on average, half the size of the original studies (see also Camerer et al., 2018; Hawkins et al., 2018). Similarly, Camerer et al. (2016) replicated 18 experiments in economics and found numerically smaller effect sizes in 14 of them, compared to the results of the original experiments. Although some (less than half) of the effects tested in Many Labs 1 (Klein et al., 2014) yielded larger effects than the originals, 
Many Labs 3 (Ebersole et al., 2016) found systematically smaller effect sizes in all cases, and so have all the Registered Replication Reports (RRR) published so far in Perspectives in Psychological Science (e.g., Hagger et al., 2016; O’Donnell et al., 2018; Wagenmakers et al., 2016)

Many factors may explain why observed effect sizes tend to decline over time. In some cases, the decline may reflect a genuine change in the prevalence or strength of the effect. For instance, meta-analytic evidence that the effectiveness of cognitive behavioral therapy is falling has been attributed to a decrease in the placebo effect elicited by this type of therapy (Johnsen \& Friborg, 2015). In the same vein, Dijksterhuis (2018) and Strack (2016) have argued that failures to replicate their findings in RRRs are possibly due to the fact that participants in these studies, usually psychology students, are now more likely to be familiar with the effects under study, as they have become common topics in undergraduate psychology courses (see also Rand, 2018, for a similar argument). It is also possible that the smaller effects reported in most replication attempts are due to the use of imperfect methods. For instance, it has been claimed that "replicators" may lack the necessary expertise or competence (Baumeister, 2016), that the procedures of multi-site studies may be less sensitive than those of the original studies (Baumeister \& Vohs, 2016), and that the samples tested in replications may differ in crucial characteristics from the samples tested in the original studies (Gilbert, King, Pettigrew, \& Wilson, 2016; Vohs, 2015).

These criticisms notwithstanding, in some cases the larger-than-usual effects reported in seminal studies may simply be the cumulative product of different biases inherent to the process of scientific discovery. Given that the most prestigious scientific journals are typically unwilling to publish studies with non-significant results, the published record offers a biased sample of all the studies that researchers have conducted 
on a given topic and of all the analyses that researchers have conducted on their datasets (Franco, Malhotra, \& Simonovits, 2014; Rosenthal, 1979; Sterling, 1959). As a result of selection on statistical significance, the effect sizes of published studies usually misrepresent and exaggerate the true size of the underlying effects (Ioannidis, 2008). Unsurprisingly, follow-up studies using similar methods will tend to find smaller effects, sometimes even failing to reach statistical significance. In these cases, the subsequent decline will be more evident in studies that rely on more powerful methods (i.e., larger samples, more reliable measures) and that adhere to more transparent reporting standards (i.e., pre-registered studies, open data), as these studies are more likely to detect and report unbiased effect sizes. In a particularly clear example of this trend, a recent study by Kaplan and Irvin (2015) showed that the number of positive results in large clinical trials funded by the National Heart, Lung, and Blood Institute has declined steadily after year 2000, following a regulation change that made pre-registration compulsory for all projects funded by this institution. In general, pre-registered trials tend to report smaller effects than unregistered trials (Papageorgiou, Xavier, Cobourne, \& Eliades, 2018).

\section{Decline effects and ego depletion}

Readers of this special issue on ego depletion will quickly recognize that decline effects are particularly relevant for this area of research. Since the publication of the seminal papers on ego depletion more than twenty years ago (Baumeister, Bratslavsky, Muraven, \& Tice, 1998; Muraven, Tice, \& Baumeister, 1998), hundreds of studies have consistently reported that performance in a task demanding self-control is hindered by previous acts of self-control. An influential meta-analysis of 83 studies comprising almost two hundred independent tests concluded that ego depletion was a robust, medium-to-large effect (Hagger, Wood, Stiff, \& Chatzisarantis, 2010). Until five years ago, it would have 
seemed absurd to question the robustness of these findings. And yet, one of the most recent reviews on this effect is titled "Is ego depletion real?" (Friese, Loschelder, Gieseler, Frankenbach, \& Inzlicht, in press). What has happened over these few years to justify this sudden change of mind?

Between 2013 and 2015, Evan Carter and colleagues published a series of metaanalytic studies showing alarming signs of publication and reporting biases in the egodepletion literature (Carter, Kofler, Forster, \& McCullough, 2015; Carter \& McCullough, 2013, 2014). Several analyses converged to the conclusion that once corrected for bias, the average effect size of these studies might not be significantly different from zero (see also Yost, 2016). To dissipate all doubts about the reliability of these effects, Martin Hagger and Nikos Chatzisarantis coordinated a large-scale RRR collecting data from 23 independent laboratories which, disappointingly, failed to find convincing evidence of ego depletion (Hagger et al., 2016). Baumeister and Vohs (2016) argued that the null results could be attributable to the unfortunate choice of a low-demanding depleting task -a criticism supported by complementary analyses by Dang (2016) and Drummond and Philipp (2017)- and that no safe conclusion about the reliability of ego-depletion effects could be drawn from a single RRR exploring just one among many alternative procedures. Following up on this criticism, Kathleen Vohs, Brandon Schmeichel, and Roy Baumeister have designed and coordinated a second RRR. Although while I write these lines the final results of this RRR are not formally published, according to a preliminary presentation at the 2018 Annual Convention of the Society for Personality and Social Psychology the study found a statistically significant, but tiny $(d=0.08)$, effect (Srivastava, 2018). While it is legitimate to see this result as a "successful" demonstration of ego depletion, in the sense that the effect is non-zero according to null hypothesis significance testing, it is also clear that this effect is remarkably smaller than the early demonstrations of the effect. 
The goal of the present study is to explore in detail the decline of depletion effects since the earliest demonstrations of this phenomenon. Based on the literature reviewed in the previous paragraphs, the reader may think that the decline of depletion effects is a recent trend driven just by the disappointing results of the two RRRs described above. On the contrary, the following analyses suggest that ego depletion has been declining progressively over the last two decades and that, in a way, the results of the two RRRs were entirely "predictable" on the basis of the evidence available since at least 2010 . As explained above, decline effects can arise for many different reasons, ranging from true changes in the state of the world to biases in the process of scientific research. Consistent with the latter interpretation, the distribution of results across time suggests that one of the reasons why depletion effects are smaller now than they were twenty years ago is that, in general, recent studies have tended to rely on larger samples, presumably leading to the publication of more precise (and less biased) effect size estimates. At the same time, the publication system has become increasingly tolerant with the publication of null replications of ego depletion, making recent research findings less impressive but perhaps more believable than earlier demonstrations of ego depletion.

\section{Method}

\section{Data sets from previous meta-analyses}

To explore decline effects in the ego depletion literature I reanalyzed data from the three most comprehensive meta-analyses conducted so far: Hagger et al. (2010), Carter et al. (2015), and Dang (2018). Beyond the fact that the meta-analysis conducted by Hagger et al. is older and does not include the most recent research, the three meta-analyses also differ regarding the number of included studies and the study selection criteria. Specifically, Carter et al. limited their meta-analyses to studies relying on frequently used 
manipulation tasks and frequently used outcome tasks, which resulted in a smaller set of studies $(k=118)$ compared to Hagger et al. $(k=198)$. In addition, Carter et al. included "grey literature" in their analyses, while the meta-analysis conducted by Hagger et al. was entirely based on published research. The dataset gathered by Dang overlaps substantially with that of Carter et al., but it includes more recent studies that were not available at the time Carter et al. conducted their literature search, resulting in a somewhat larger set of studies $(k=142)$.

To conduct the following analyses, I extracted information about the individual studies included in each meta-analysis from the supplementary files published by Hagger et al. (2010), Carter et al. (2015), and Dang (2018). In the case of Hagger et al., I extracted the effect sizes of each individual study from Appendix B. Given that the table reporting this information only included absolute sample sizes, I assumed that both the control and the experimental groups had equal sample sizes. If the absolute sample size was an odd number, I assumed that the experimental group had one more participant than the control group. Data from Carter et al. were extracted from the .RData files publicly available in the supplemental materials. In this case, the authors provided exact sample sizes for each experimental condition. Finally, all the relevant data from Dang were extracted from the Excel file included in the supplemental material. Given that this data file did not include information about the publication status (published vs. unpublished) of each study, I took this information from Table 1 in the supplemental materials and from Carter et al. for the older studies included in both meta-analyses. All the information that I extracted from these three meta-analyses is available in three .csv files at https://osf.io/w4ufj/. 
In addition to reanalyzing data from previous systematic reviews, I also searched for new studies published since 2016 that were not included in those meta-analyses. Specifically, on September 28, 2018, I conducted a literature search on the Web of Science using the same search terms employed by Carter et al. This returned 1,030 articles published since 2016. These articles were then screened on the basis of the information contained in the titles, abstracts and, when necessary, main texts to identify studies that met the inclusion criteria used by Carter et al. This led to the selection of 17 articles containing at least one valid study. Two of these did not report sufficient information to compute effect sizes and their authors did not reply to email requests. For the rest of articles, this information was available in the manuscript itself of sent by the authors upon request. In total, I was able to compute 20 independent effect sizes from the selected articles.

In addition to these, I also included the large-scale registered replication conducted by Hagger et al. (2016). Note that, in principle, this study does not meet the selection criteria used by Carter et al. (2015), because in that meta-analysis only studies using frequent outcome tasks were included. Unfortunately, the multi-source interference task used by Hagger et al. (2016) is not one of them. However, given that this study represents a landmark in the recent history of ego-depletion research and that the study comprised 23 independent replications of the effect, I decided to include it in the following analyses. For consistency with this decision, I also included the study by Sripada, Kessler, and Jonides (2014), on which the registered replication report was based, and a recent study by Emmerling et al. (2017) that relied on the same outcome task. Following the recommendations of the reviewers of an earlier draft of this article, I also included in the updated analyses two pre-registered studies by Dang, Liu, Liu, and Mao (2017) and 
Garrison, Finley, and Schmeichel (in press), although some of the studies reported in these papers do not comply with the selection criteria of Carter et al.

As in Carter et al. (2015) and Dang (2018) I used Hedges' $g$ as an effect size estimate. Unlike previous meta-analyses, in the updated literature search I also included studies that manipulated ego depletion within-subjects, exposing each participant to both the depletion and control conditions on different sessions. In these cases, I computed effect sizes using the standard deviation of the control condition to standardize mean differences. For the computation of effect size variances, I followed the guidelines of Morris and DeShon (2002), assuming a correlation of $r=.50$ among repeated measures when this information was not available in the articles. As in Carter et al., for experiments in which an individual difference variable was hypothesized to moderate the effect of ego depletion, I only included in the analyses the main effect of ego depletion. Similarly, as in Carter et al. and Hagger et al. (2010), for studies that manipulated a variable hypothesized to moderate the effect, I only included the level of the moderator not thought to attenuate the depletion effect. Further information about the selection of studies and computation of effect sizes is available at https://osf.io/ms3a7/.

\section{Data analysis}

All the statistical analyses were conducted in R. In meta-analytic research it is common to weight studies differently depending on their precision. This means that experiments comprising large samples of participants are weighted more heavily than smaller studies. Following this logic, unless otherwise noted, all the regressions reported in the Results section are weighted-least squares (WLS) regressions, weighting each study by the inverse of its effect size variance. All the scripts used to produce the following figures and results are publicly available at https://osf.io/hmqvd/. 
Given that the substantial overlap between the set of studies meta-analysed by Carter et al. (2015) and Dang (2018), in the present article I only report analyses for the latter data set. However, the scripts available at the OSF include also analyses for the data set gathered by Carter et al.

\section{Results}

Figures 1A and 1B plot effect sizes against year of publication for the studies metaanalysed by Hagger et al. (2010) and by Dang (2018), respectively, together with the bestfitting WLS regressions. The slopes were statistically significant in both cases, $b=-0.029$, $p=.007$; and $b=-0.029, p=.001$, respectively. An otherwise identical WLS regression excluding unpublished studies from the Dang data set also yielded a significant slope, $b=$ $0.024, p=.014$, suggesting that the downward trend is not driven by the inclusion of grey literature. Figure $1 \mathrm{C}$ shows the results obtained with the updated data set. Again, the slope was statistically significant, both including, $b=-0.033, p<.001$, and excluding, $b=$ $0.037, p<.001$, the unpublished studies selected by Dang. Excluding three "mega-studies" with more than 1,000 participants (Hagger et al., 2016; Singh \& Göritz, 2018; Vadillo, Gold, \& Osman, 2018) did not change the results either, $b=-0.027, p<.001$. According to the most recent dataset and considering all the studies, the best regression line crosses the $g$ $=0$ axis at year 2020 .

An interesting pattern of the results depicted in Figure 1 is that the most recent studies seem to rely on substantially larger samples (represented as larger circles). This is more clearly seen in Figure 2, where (log) sample sizes are plotted against year of publication for the updated data set. The slope of the WLS regression relating both variables is statistically significant, $b=0.227, p<.001$. The slope is also significant if "mega-studies" with more than 1,000 participants are removed from the analysis, $b=$ 
$0.105, p<.001$, and also using an ordinary least squares regression (i.e., if larger studies are not weighted more heavily), $b=0.048, p<.001$. Although not shown in Figure 2 , the progressive increase of effect sizes is also present in the data sets analysed by Hagger et al. (2010), $b=0.014, p<.001$, and by Dang (2018), $b=0.031, p=.017$.

Furthermore, the analysis of the updated data set shows that sample sizes are also related to effect sizes, as shown by the significant slope of the WLS regression of effect sizes on log sample sizes, $b=-0.095, p<.001$. The negative correlation between sample size (or study precision) and effect sizes has already been reported in Carter et al.'s (2014) reanalysis of Hagger et al. (2010) and in the more recent meta-analysis of Carter et al. (2015). With some caveats (see Sterne et al., 2011), this negative relationship could indicate publication or reporting biases.

The fact that sample sizes have increased over time and that larger studies yield smaller effects suggests that sample size may be a mediating factor in the decline of depletion effects. To test this hypothesis, I conducted an additional WLS regression predicting effect sizes from log sample sizes and year of publication. The comparison of this analysis and the ones reported above showed that including log sample size in the model reduced the slope of year from -0.033 to -0.021 . Sobel's test for mediation was statistically significant, $z=-2.447, p=.014$, confirming that sample size mediates the relationship between year of publication and effect size. Note, however, that the mediation was only partial, as year of publication still accounted for a significant proportion of variance in effect sizes after controlling for the effects of sample size, $p=.003$. In other words, the progressive increase of sample sizes explains a significant proportion of the decline in depletion effects, although other factors are probably contributing as well.

In light of the data presented in Figures $1 \mathrm{~A}-1 \mathrm{C}$, it is tempting to think that many of the small effect sizes reported over the last decade must have failed to reach statistical 
significance. To test this impression, I divided all effect sizes in the updated data set by their standard errors to obtain a $z$-score for each study. If the absolute value of the $z$-score was larger than 1.96, I classified that effect size as statistically significant. Figure 3 shows the distribution of significant and non-significant studies over time. As can be seen, there is a progressive increase in the absolute number and relative proportion of non-significant results. A weighted logistic regression confirmed that the proportion of significant results was lower among latter studies, $b=-0.186, p<.001$, and this result held even if unpublished studies from the Dang dataset were removed from the analysis, $b=-0.204, p<$ .001 . One of the signs of bias detected by Carter et al. $(2014,2015)$ was that the proportion of statistically significant results in this literature was higher than the average statistical power of studies, that is to say, that the results were "too good to be true" (Francis, 2012). The data depicted in Figure 3 suggest that this bias has been systematically decreasing over time since at least 2008, well before the onset of the "replication crisis" and Carter et al.'s critical reappraisal of this literature.

An interesting novelty in psychological research is that the replication crisis has led to the adoption of more transparent scientific standards, including the pre-registration of hypotheses, methods and analytic procedures. Although only one of the studies included in Dang's (2018) meta-analysis was pre-registered (Lurquin et al., 2016), the updated data set contains four additional pre-registered studies (Dang et al., 2017; Garrison et al., in press; Hagger et al., 2016). Previous analyses have suggested that the proportion of nonsignificant results is larger among registered reports than among traditional scientific papers (Allen \& Mehler, 2018; Kaplan \& Irvin, 2015; Papageorgiou et al., 2018). Bearing this in mind, it is possible that an additional factor shaping the decline of depletion effects is that the pre-registered studies published since 2016 are pulling effect sizes down. 
Figure 4 shows the effect sizes of pre-registered and non-registered studies, together with meta-analytic averages. In agreement with the hypothesis that pre-registered studies tend to yield smaller effects, Figure 4 suggests that the meta-analytic averages of preregistered studies are somewhat lower than their non-registered counterparts. Moderator analyses reveal that the difference between registered and non-registered studies is significant in the fixed-effect model, $Q(1)=10.53, p=.001$, but not in the random-effects model, $Q(1)=2.12, p=.145$.

\section{Discussion and Concluding Comments}

The fact that ego-depletion effects have declined over the last two decades does not necessarily mean that they will continue to do so in the future and, if that happens, most certainly the decay will not follow a linear trend. There is no reason to think that we will see the twilight of ego depletion early in the morning of April $15^{\text {th }}, 2020$, as predicted by the regression line in Figure 1C, and that the effect will then become increasingly negative over the years to follow. Assuming that the most recent studies are as unbiased and accurate as they can possibly be (i.e., highly powered, preregistered), we can expect effect sizes to plateau around small but positive values. Alternatively, if other sources of bias are still present even among the best studies (e.g., unsuccessful blinding of experimenters and participants, conceptual confounds), we can expect that the effect will continue to decline and perhaps disappear as these biases are further corrected in future studies.

Even if the best estimate of depletion effects eventually converges to a small but nonzero value, the landscape of ego depletion research and theory may change beyond recognition in just a few years. As noted by Carter and McCullough (2018), if the true effect size of ego depletion turns out to be, for instance, $d=0.15$, then a simple two-group experiment will need almost 1,400 participants to reach a statistical power of $80 \%$. The 
figure grows to almost 5,600 for an experiment planning to explore an attenuated interaction (Simonsohn, 2014). In the end, research on ego depletion may disappear not because the effect does not exist, but because the sample sizes required to explore such small effects are simply beyond the reach of most researchers.

The present study not only shows that depletion effects have been declining progressively and that this trend was predictable since at least 2010, it also provides some hints to understand the mechanisms that have driven this process. Sample sizes have become progressively larger over the last two decades (Figure 2) and small and null findings have gained more visibility (Figure 3). Both factors contribute to making research on ego depletion more robust to publication and reporting biases. Taken collectively, these results suggest that early demonstrations of ego depletion were grossly inflated by selective reporting or other sources of bias and that the literature was slowly correcting itself by 2010. The meta-analytic work published in 2014 and 2015 (Carter et al., 2014, 2015), with the additional momentum provided by the replication crisis (Pashler \& Wagenmakers, 2012) and RRRs (Simons, Holcombe, \& Spellman, 2014), has speeded up the process to such an extent that current estimates of depletion effects are practically indistinguishable from zero.

In the biomedical literature, there is clear evidence that studies with non-significant results take longer to publish (Ioannidis, 1998; Stern \& Simes, 1997). It is possible that ego-depletion researchers have been reluctant or unable to publish their non-significant results until ten years ago. With the advent of online mega-journals, it has become substantially easier to publish studies with unclear or non-significant results, given that many of these journals explicitly encourage reviewers to assess papers on the basis of methodological rigor and not of statistical significance. Although none of the papers reviewed by Hagger et al. (2010) were published in journals like PLoS One (published 
since 2006) or Frontiers in Psychology (published since 2010), five of the papers included in the meta-analysis of Dang (2018) were published in these outlets and five additional papers included in the updated data set were published in these two journals or in the recently funded Royal Society Open Science. In a world where articles are assessed on the basis of methodological standards and statistical results do not have to be spectacular to get published, effect sizes will logically be modest, but more representative of the true state of nature.

Additionally, studies with null results or small effects may have become more surprising -and therefore more informative- after ego depletion became an established effect. A long tradition of research in learning and memory posits that the amount of learning stimulated by an event heavily depends on the extent to which it conflicts with previous knowledge (Clark, 2013; Rescorla \& Wagner, 1972). Perhaps scientific progress is not very different from human learning: Failures to replicate an effect are only noteworthy if there were reasons to expect the effect to begin with. If follows from this that journals and scientists will pay little attention to studies with null results at early stages of research about a topic. From this point of view, decline effects would indicate nothing but the eventual correction of the bias produced by our natural tendency to focus on surprising and unexpected events.

Of course, although the present study suggests that the higher precision of recent studies and journals' increasing tolerance to non-significant findings have played a role in the decline effect, it is likely that other factors may have contributed to this process. For instance, it is not impossible that self-control has increased over time and that, consequently, participants tested in ego-depletion experiments are now more efficient at inhibiting automatic responses than two decades ago. Meta-analytic evidence does suggest that children are more able to delay gratification in the famous Marshmallow test now than 
50 years ago (Protzko, 2017). It seems unlikely, though, that this small, long-term increase in self-control can fully account for the trends explored in the present analysis, as it fails to explain why the evidence of bias has weakened in the last decade.

To sum up, the present study shows (a) that ego-depletion effects have been declining systematically over the last two decades and (b) that this process began well before any of the Registered Replication Reports on ego depletion were conceived and even before Evan Carter and colleagues published their studies on publication bias in this domain. It also suggests (c) that the decline is, at least partly, caused by the fact that recent studies tend to rely on larger samples and, therefore, yield more accurate effect sizes estimates, and (d) that the selective publication of significant findings has become less prevalent over the course of the two decades of research on ego depletion. The implications for future research are clear: If the factors that drove this decline in the past continue to exert their action in the near future, we should get used to very small effect sizes in the ego-depletion literature and perhaps prepare for the eventual disappearance of the effect by 2020 . 


\section{References}

Allen, C. P. G., \& Mehler, D. M. A. (2018). Open Science challenges, benefits and tips in early career and beyond. Unpublished manuscript. Available at https://doi.org/10.31234/osf.io/3czyt

Baumeister, R. F. (2016). Charting the future of social psychology on stormy seas: Winners, losers, and recommendations. Journal of Experimental Psychology, 66, 153-148.

Baumeister, R. F., Bratslavsky, E., Muraven, M., \& Tice, D. M. (1998). Ego depletion: Is the active self a limited resource? Journal of Personality and Social Psychology, 74, $1252-1265$.

Baumeister, R. F., \& Vohs, K. D. (2016). Misguided effort with elusive implications. Perspectives on Psychological Science, 11, 574-575.

Camerer, C. F., Dreber, A., Forsell, E., Ho, T. H., Huber, J., Johannesson, M., ... \& Wu, H. (2016). Evaluating replicability of laboratory experiments in economics. Science, $351,1433-1436$.

Camerer, C. F., Dreber, A., Holzmeister, F., Ho, T.-H., Huber, J., Johannesson, M., ... \& Wu, H. (2018). Evaluating the replicability of social science experiments in Nature and Science between 2010 and 2015. Nature Human Behaviour, 2, 637-644.

Carter, E. C., Kofler, L. M., Forster, D. E., \& McCullough, M. E. (2015). A series of metaanalytic tests of the depletion effect: Self-control does not seem to rely on a limited resource. Journal of Experimental Psychology: General, 144, 796-815.

Carter, E. C., \& McCullough, M. E. (2013). Is ego depletion too incredible? Evidence for the overestimation of the depletion effect. Behavioral and Brain Sciences, 36, 683684. 
Carter, E. C., \& McCullough, M. E. (2014). Publication bias and the limited strength model of self-control: Has the evidence for ego depletion been overestimated? Frontiers in Psychology, 5, 823.

Carter, E. C., \& McCullough, M. E. (2018). A simple, principled approach to combining evidence from meta-analysis and high-quality replications. Advances in Methods and Practices in Psychological Science, 1, 174-185.

Clark, A. (2013). Whatever next? Predictive brains, situated agents, and the future of cognitive science. Behavioral and Brain Sciences, 36, 181-204.

Dang, J. (2016). Commentary: “A multilab preregistered replication of the ego-depletion effect”. Frontiers in Psychology, 7, 1155.

Dang, J. (2018). An updated meta-analysis of the ego depletion effect. Psychological Research, 82, 645-651.

Dang, J., Liu, Y., Liu, X., \& Mao, L. (2017). The ego could be depleted, providing initial exertion is depleting. Social Psychology, 48, 242-245.

Dijksterhuis, A. (2018). Reflection on the professor priming replication report.

Perspectives on Psychological Science, 13, 295-296.

Drummond, A., \& Philipp, M. C. (2017). Commentary: "Misguided effort with elusive implications" and "A multi-lab pre-registered replication of the ego depletion effect". Frontiers in Psychology, 8, 273.

Ebersole, C. R., Atherton, O. E., Belanger, A. L., Skulborstad, H. M., Allen, J. M., Banks, J. B. ... \& Nosek, B. A. (2016). Many Labs 3: Evaluating participant pool quality across the academic semester via replication. Journal of Experimental Social Psychology, 67, 68-82.

Emmerling, F., Martijn, C., Alberts, H. J. E. M., Thomson, A. C., David, B., Kessler, D., Schuhmann, T., \& Sack, A. T. (2017). The (non-)replicability of regulatory resource 
depletion: A field report employing non-invasive brain stimulation. PLoS ONE, 12, e0174331.

Francis, G. (2012). Too good to be true: Publication bias in two prominent studies from experimental psychology. Psychonomic Bulletin \& Review, 19, 151-156.

Franco, A., Malhotra, N., \& Simonovits, G. (2014). Publication bias in the social sciences: Unlocking the file drawer. Science, 345, 1502-1505.

Friese, M., Loschelder, D. D., Gieseler, K., Frankenbach, J., \& Inzlicht, M. (in press). Is ego depletion real? An analysis of arguments. Personality and Social Psychology Review.

Garrison, K. E., Finley, A. J., \& Schmeichel, B. J. (in press). Ego depletion reduces attention control: Evidence from two high-powered preregistered experiments. Personality and Social Psychology Bulletin.

Gehr, B. T., Weiss, C., \& Porzsolt, F. (2006). The fading of reported effectiveness: A meta-analysis of randomised controlled trials. BMC Medical Research Methodology, 6, 25 .

Gilbert, D. T., King, G., Pettigrew, S., \& Wilson, T. D. (2016). Comment on "Estimating the reproducibility of psychological science". Science, 351, $1037 \mathrm{~b}$.

Hagger, M. S., Chatzisarantis, N. L. D., Alberts, H., Angonno, C. O., Batailler, C., Birt, A., ... \& Zwienenberg, M. (2016). A multi-lab pre-registered replication of the egodepletion effect. Perspectives on Psychological Science, 11, 546-573.

Hagger, M. S., Wood, C., Stiff, C., \& Chatzisarantis, N. L. (2010). Ego depletion and the strength model of self-control: A meta-analysis. Psychological Bulletin, 136, 495525. 
Hawkins, R. X. D., Smith, E. N., Au, C., Arias, J. M., Catapano, R., Hermann, E., ... \& Frank, M. C. (2018). Improving the replicability of psychological science through pedagogy. Advances in Methods and Practices in Psychological Science, 1, 7-18.

Ioannidis, J. P. A. (1998). Effect of the statistical significance of results on the time to completion and publication of randomized efficacy trials. Journal of the American Medical Association, 279, 281-286.

Ioannidis, J. P. A. (2008). Why most discovered true associations are inflated. Epidemiology, 19, 640-648.

Jennions, M. D., \& Moller, A. P. (2002). Relationships fade with time: A meta-analysis of temporal trends in publication in ecology and evolution. Proceedings of the Royal Society of London, B, 269, 43-48.

Johnsen, T. J., \& Friborg, O. (2015). The effects of cognitive behavioral therapy as an antidepressive treatment is falling: A meta-analysis. Psychological Bulletin, 14, 747-768.

Kaplan, R. M., \& Irvin, V. L. (2015). Likelihood of null effects on large NHLBI clinical trials has increased over time. PLoS ONE, 10, e0132382.

Klein, R. A., Ratliff, K. A., Vianello, M., Adams, R. B., Jr., Bahnik, S., Bernstein, M. J., ... \& Nosek, B. A. (2014). Investigating variation in replicability: A “many labs" replication project. Social Psychology, 45, 142-152.

Lehrer, J. (2011, December 13). The truth wears off: Is there something wrong with the scientific method? New Yorker Magazine, pp. 52-56.

Lurquin, J. H., Michaelson, L. E., Barker, J. E., Gustavson, D. E., von Bastian, C. C., Carruth, N. P., \& Miyake, A., (2016). No evidence of the ego-depletion effect across task characteristics and individual differences: A pre-registered study. PLoS ONE, 11, e0147770. 
Morris, S. B., \& DeShon, R. P. (2002). Combining effect size estimates in meta-analysis with repeated measures and independent-groups designs. Psychological Methods, 7 , 105-125.

Muraven, M., Tice, D. M., \& Baumeister, R. F. (1998). Self- control as a limited resource: Regulatory depletion patterns. Journal of Personality and Social Psychology, 74, 774-789.

O’Donnell, M., Nelson, L. D., Ackermann, E., Aczel, B., Akhtar, A., Aldrovandi, S., ... \& Zrubka, M. (2018). Registered Replication Report: Dijksterhuis \& van Knippenberg (1998). Perspectives on Psychological Science, 13, 268-294.

Open Science Collaboration. (2015). Estimating the reproducibility of psychological science. Science, 349(6251), aac4716.

Papageorgiou, S. N., Xavier, G. M., Cobourne, M. T., \& Eliades, T. (2018). Registered trials report less beneficial treatment effects than unregistered ones: A metaepidemiological study in orthodontics. Journal of Clinical Epidemiology, 100, 44-52.

Pashler, H., \& Wagenmakers, E. J. (2012). Editors' introduction to the special section on replicability in psychological science: A crisis of confidence? Perspectives on Psychological Science, 7, 528-530

Pereira, T. V., Horwitz, R. I., \& Ioannidis, J. P. A. (2012). Empirical evaluation of very large treatment effects of medical interventions. JAMA, 308, 1676-1684.

Protzko, J. (2017, September 13). Kids these days. Retrieved from osf.io/j9tuz Protzko, J., \& Schooler, J. W. (2017). Decline effects: Types, mechanisms, and personal reflections. In S. O. Lilienfeld \& I. D. Waldman (Eds.), Psychological science under scrutiny: Recent challenges and proposed solutions (pp. 85-107). Chichester, West Sussex, UK: John Wiley \& Sons, Inc. 
Rand, D. G. (2018). Non-naivety may reduce the effect of intuition manipulations. Nature Human Behavior, 2, 602.

Rescorla, R. A., \& Wagner, A. R. (1972). A theory of Pavlovian conditioning: Variations in the effectiveness of reinforcement and nonreinforcement. In A. H. Black \& W. F. Prokasy (Eds.), Classical conditioning II (pp. 64-99). New York, NY: AppletonCentury-Crofts.

Rosenthal, R. (1979). The file drawer problem and tolerance for null results. Psychological Bulletin, 86, 638-641.

Schooler, J. (2011). Unpublished results hide the decline effect. Nature, 470, 437.

Simmons, L. W., Tomkins, J. L., Kotiaho, J. S., \& Hunt, J. (1999). Fluctuating paradigm. Proceedings of the Royal Society of London, B, 266, 593-595.

Simons, D. J., Holcombe, A. O., \& Spellman, B. A. (2014). An introduction to registered replication reports at Perspectives on Psychological Science. Perspectives on Psychological Science, 9, 552-555

Simonsohn, U. (2014). No-way interactions [Blog post]. Retrieved from http://datacolada.org/17

Singh, R. K., \& Göritz, A. S. (2018). Ego depletion does not interfere with working memory performance. Frontiers in Psychology, 9, 538.

Sripada, C., Kessler, D., \& Jonides, J. (2014). Methylphenidate blocks effort-induced depletion of regulatory control in healthy volunteers. Psychological Science, 25, $1227-1234$

Srivastava, S. [@hardsci]. (2018, March 3). About to start - a symposium session on fresh* results from a multi-site ego depletion study \#spsp2018 [Tweet]. Retrieved from https://twitter.com/hardsci/status/970015349499465729 
Sterling, T. D. (1959). Publication decisions and their possible effects on inferences drawn from tests of significance -or viceversa. Journal of the American Statistical Association, 54, 30-34.

Stern, J. M., \& Simes, R. J. (1997). Publication bias: Evidence of delayed publication in a cohort study of clinical research projects. British Medical Journal, 315, 640-645.

Sterne, J. A. C., Sutton, A. J., Ioannidis, J. P. A., Terrin, N., Jones, D. R., Lau, J., ... Higgins, J. P. (2011). Recommendations for examining and interpreting funnel plot asymmetry in meta-analyses of randomised controlled trials. British Medical Journal, 343, d4002.

Strack, F. (2016). Reflection on the smiling registered replication report. Perspectives on Psychological Science, 11, 929-930.

Trikalinos, T. A., Churchill, R., Ferri, M., Leucht, S., Tuunainen, A., Wahlbeck, K., Ioannidis, J. P. A. (2004). Effect sizes in cumulative meta-analyses of mental health randomized trials evolved over time. Journal of Clinical Epidemiology, 57, 11241130.

Trikalinos, T. A., \& Ioannidis, J. P. A. (2005). Assessing the evolution of effect sizes over time. In H. R. Rothstein, A. J. Sutton, \& M. Borenstein (Eds.), Publication bias and meta-analysis: Prevention, assessment and adjustments (pp. 241-259). New York: John Wiley \& Sons.

Vadillo, M. A., Gold, N., \& Osman, M. (2018). Searching for the bottom of the ego well: Failure to uncover ego depletion in Many Labs 3. Royal Society Open Science, 5, 180390.

Vohs, K. D. (2015). Money priming can change people's thoughts, feelings, motivations, and behaviors: An update on 10 years of experiments. Journal of Experimental Psychology: General, 144, e86-e93. 
Wagenmakers, E.-J., Beek, T., Dijkhoff, L., Gronau, Q. F., Acosta, A., Adams, R. B., Jr., ... \& Zwaan, R. A. (2016). Registered Replication Report: Strack, Martin, \& Stepper (1988). Perspectives on Psychological Science, 11, 917-928.

Yost, T. A. (2016). Meta-analytic and empirical estimates of the resource depletion effect size. Unpublished doctoral dissertation, University of Minnesota. 


\section{Figure Captions}

Figure 1. Panels A and B plot the standardized effect sizes of the meta-analyses conducted by Hagger et al. (2010), Dang (2018), respectively, against the year of publication of the primary studies. The size of each circle is inversely proportional to the study's variance. Panel C contains additional studies published between 2016 and 2018.

Figure 2. Sample sizes of each study included in the updated data set plotted against year of publication, with best-fitting regression lines for different subsets of studies (including vs. excluding studies with more than 1,000 participants) and different weighting criteria (weighted vs. ordinary least squares errors).

Figure 3. Evolution of studies' statistical significance over time. Full circles denote nonsignificant tests of ego depletion (absolute $\mathrm{z}>1.96$ ), while open circles denote studies with significant results. Black circles denote published studies; grey circles denote nonpublished studies ("grey literature") included in Dang' (2018) meta-analysis.

Figure 4. Comparison of effect sizes in pre-registered vs. non-registered studies. As in Figure 1, the size of each circle is inversely proportional to the study's variance. Black diamonds represent meta-analytic averages computed using a random-effects model. White diamonds represent averages computed with a fixed-effect model. 

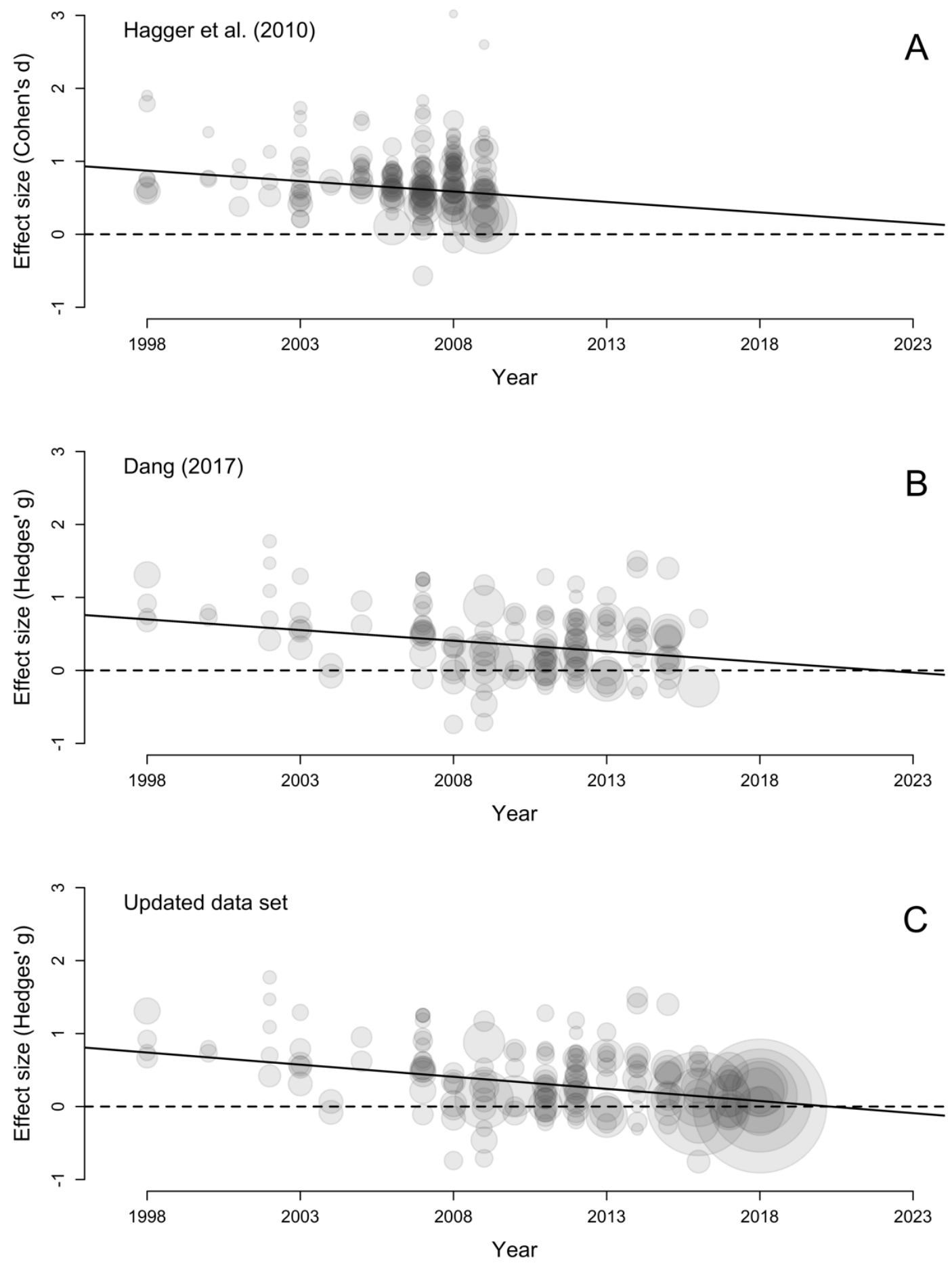

Figure \#1 


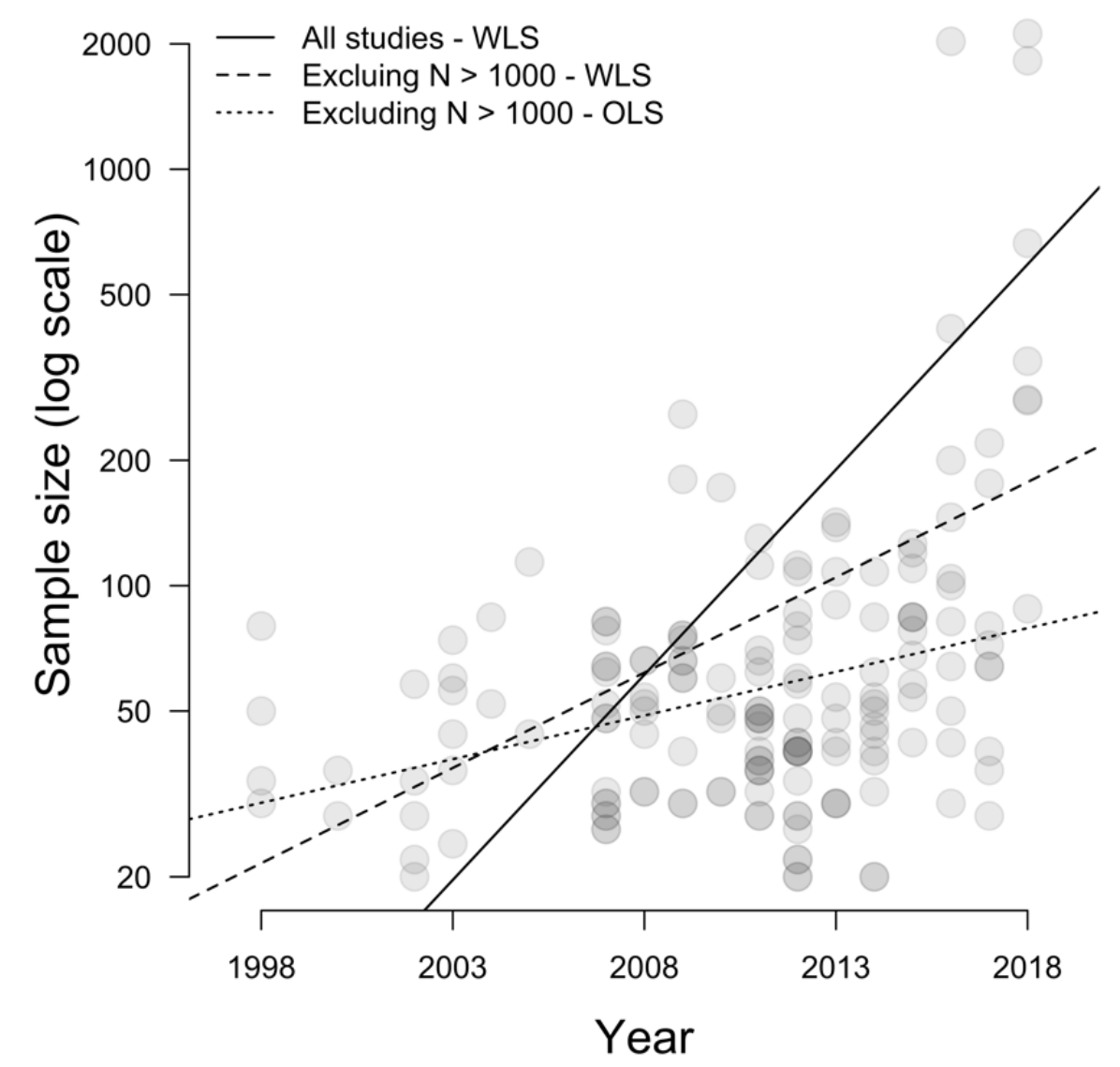

Figure \#2 
Significant

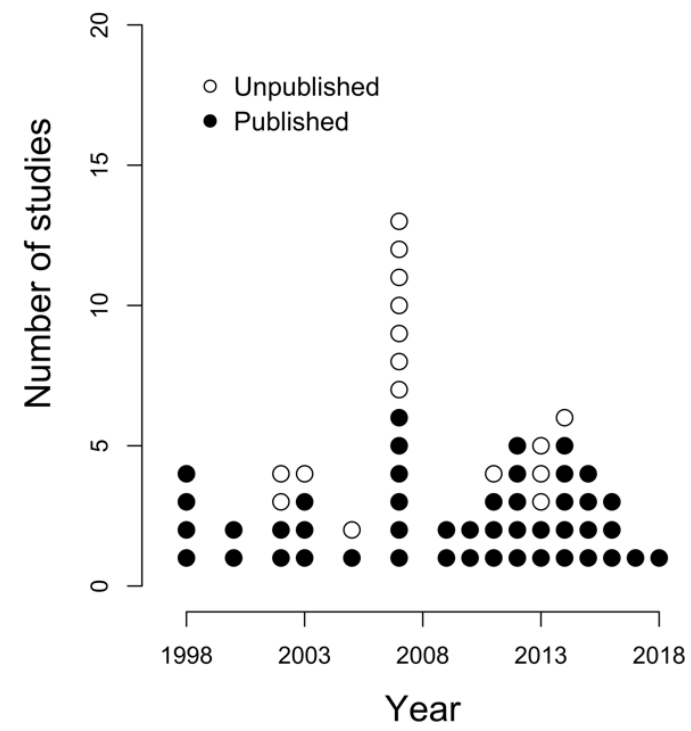

\section{Non-significant}

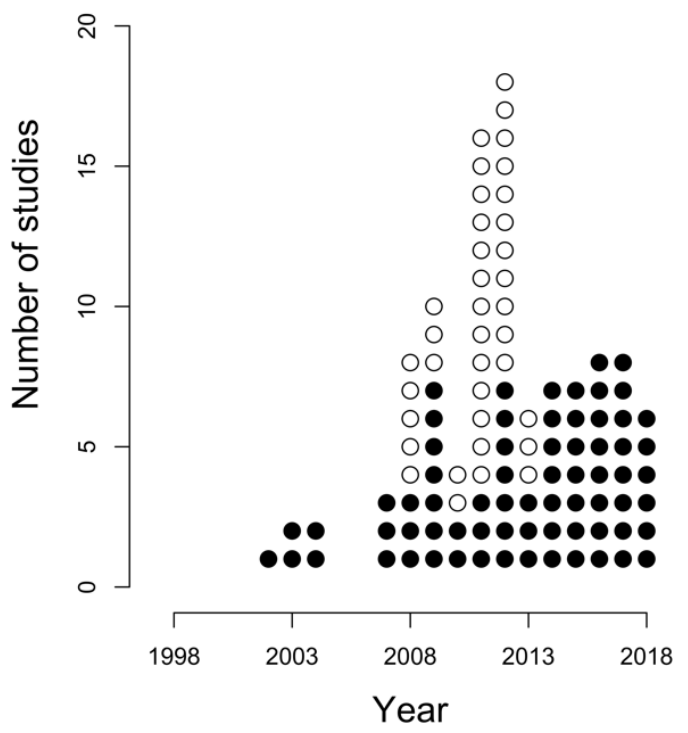

Figure \#3 


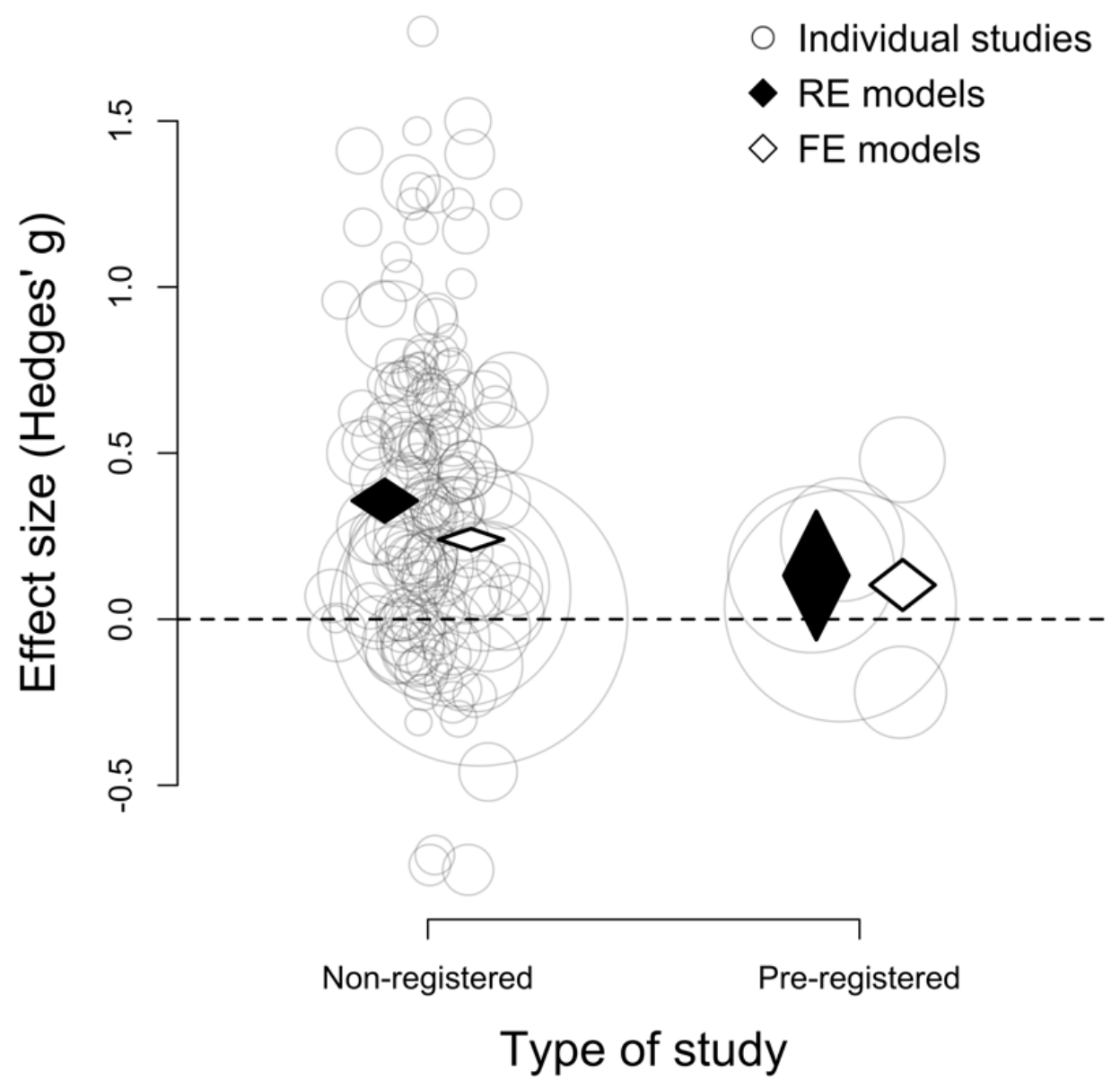

Figure \#4 